\title{
Selection of Apoptosis-Deficient Adenovirus E4orf4 Mutants in Saccharomyces cerevisiae
}

\author{
RANA AFIFI, RAKEFET SHARF, RONIT SHTRICHMAN, $\dagger$ AND TAMAR KLEINBERGER* \\ Gonda Center of Molecular Microbiology, Bruce Rappaport Faculty of Medicine, \\ Technion, Haifa 31096, Israel
}

Received 13 November 2000/Accepted 19 January 2001

\begin{abstract}
Adenovirus E4orf4 protein has been shown to induce p53-independent, protein phosphatase 2A (PP2A)dependent apoptosis in transformed cells. Furthermore, E4orf4 also induces toxicity in Saccharomyces cerevisiae in a PP2A-dependent manner (D. Kornitzer and T. Kleinberger, submitted for publication). In this work, we utilized yeast cells to select for nonapoptotic E4orf4 mutants which, in turn, were shown to possess a diminished ability to bind PP2A. The success of this selection system will provide additional apoptosis-relevant mutants for E4orf4 research and strongly supports the relevance of E4orf4-induced toxicity in $S$. cerevisiae to E4orf4-induced apoptosis in mammalian cells.
\end{abstract}

Adenovirus E4orf4 protein is a multifunctional viral regulator. Our work, as well as studies from other laboratories, has shown that the E4orf4 protein down regulates the expression of genes that have been activated by enzyme immunoassay and cyclic AMP $(3,8)$, induces hypophosphorylation of various viral and cellular proteins $(1,8)$, regulates alternative splicing of adenovirus mRNAs (1), and induces p53-independent apoptosis in transformed cells $(2,4,6,9)$. Induction of apoptosis has been reported to be caspase independent in CHO cells (4), although it is caspase dependent in other cell lines (5). We have previously shown that E4orf4 interacts with the protein phosphatase 2A (PP2A) holoenzyme through a direct association with the $\mathrm{B} \alpha / \mathrm{B} 55$ (3) or $\mathrm{B}^{\prime} / \mathrm{B} 56$ (10) subunits. We have recently shown that interaction with the $\mathrm{PP} 2 \mathrm{~A}$ complexes that include the $\mathrm{B} \alpha / \mathrm{B} 55$ subunit is required for induction of apoptosis by E4orf4 $(10,11)$. Several E4orf4 mutants have been prepared by both random and site-directed mutagenesis. These mutants have been tested for their ability to induce apoptosis and to bind PP2A (11). One mutant completely lost the ability to induce apoptosis and to bind PP2A, and a few mutants retained reduced proapoptotic and PP2A-binding capabilities. Additional nonapoptotic mutants obtained by sitedirected mutagenesis of E4orf4 conserved amino acids have also been described in a later work (7).

Recently, we demonstrated that E4orf4 induced irreversible growth arrest in Saccharomyces cerevisiae by a mechanism that required the yeast homolog of PP2A-B $\alpha, C D C 55$ (D. Kornitzer and T. Kleinberger, submitted for publication). An E4orf4 mutant (A3), which did not bind PP2A and did not induce apoptosis in mammalian cells, was also nontoxic in yeast. Our results indicated that components of the E4orf4 apoptotic pathway were conserved from yeast to mammals. Based on these results, we undertook experiments that used the yeast

\footnotetext{
* Corresponding author. Mailing address: Gonda Center of Molecular Microbiology, Bruce Rappaport Faculty of Medicine, Technion, Haifa 31096, Israel. Phone: 972-4-829-5257. Fax: 972-4-829-5225. Email: tamark@tx.technion.ac.il.

$\dagger$ Present address: Molecular, Cellular and Developmental Biology Department, University of California—Santa Barbara, Santa Barbara, CA 93106.
}

system to select additional E4orf4 mutants that have lost their proapoptotic ability. Obtaining such mutants will assist in further dissection of the underlying mechanisms of E4orf4 action.

E4orf4 DNA was cloned into a plasmid that contained the yeast $2 \mu \mathrm{m}$ origin of replication, a URA3 marker, and expressed E4orf4 from a galactose-inducible promoter. Yeast cells harboring this plasmid grew on glucose in which the galactose promoter was repressed, but did not grow on galactose in which E4orf4 expression was induced (D. Kornitzer and T. Kleinberger, submitted for publication) (Fig. 1). The E4orf4-expressing plasmid was subjected to in vitro mutagenesis by incubation at $65^{\circ} \mathrm{C}$ with $0.4 \mathrm{M}$ hydroxylamine- $0.5 \mathrm{M} \mathrm{Na}$ phosphate buffer ( $\mathrm{pH}$ 6)-1 mM EDTA, for varying lengths of time (10 to $30 \mathrm{~min}$ ). Hydroxylamine reacts with a doublestranded target DNA to create $\mathrm{N}^{4}$-hydroxycytosine, which can form a base pair with adenosine, leading to both $\mathrm{C}$ to $\mathrm{T}$ and $\mathrm{G}$ to A substitutions (12). Following dialysis against phosphatebuffered saline, cells of the widely used $S$. cerevisiae wild-type (wt) strain W303 were transformed with the mutagenized DNA and plated on glucose plates lacking uracil for $16 \mathrm{~h}$. They were then replica plated onto galactose plates lacking uracil to select for yeast cells that harbor the E4orf4-expressing plasmid but can grow on galactose. The number of transformants was estimated by growing one aliquot of transformed cells on a glucose plate. Twenty-six colonies growing on galactose were obtained from a total of 5,000 transformants. Figure 1A shows the growth of selected colonies in comparison to yeast harboring the empty vector or unmutagenized E4orf4. To discard mutants that were affected in E4orf4 expression, E4orf4 levels were measured by Western blotting. Plasmids were extracted from clones in which E4orf4 was expressed at levels similar to those of the nonmutagenized control and were used in a second cycle of transformation and selection on galactose. Nine clones that still expressed high levels of nontoxic E4orf4 (Fig. 1B) were sequenced; the results are shown in Fig. 2A.

Of the nine mutants that were obtained, seven contained a single substitution mutation. Of these, two mutations were found twice: P4L (mutants M1 and M7) and R81C (mutants M2 and M8). Mutation R81C was obtained a third time, in mutant M3, which contained a deletion of V29 as well. The 


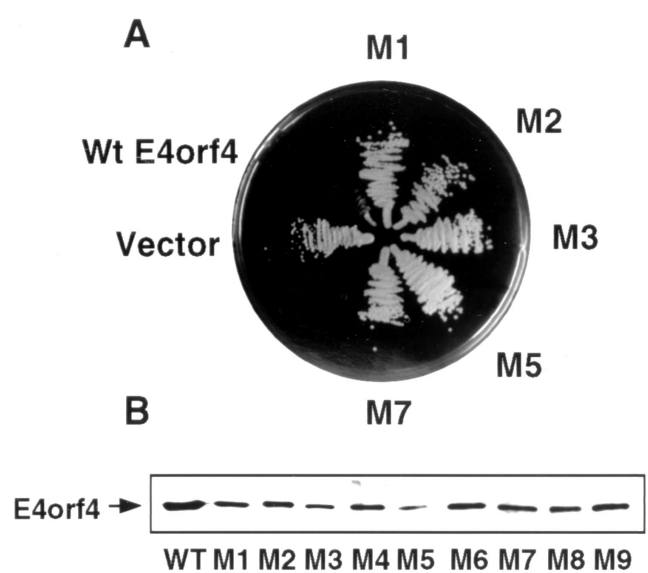

FIG. 1. Selection of mutant E4orf4 clones in yeast. (A) Yeast cells harboring the empty vector, wt E4orf4, or mutant E4orf4 cDNAs were plated on galactose and allowed to grow for 2 days. (B) E4orf4 levels in the various clones that grew on galactose were analyzed by Western blotting using E4orf4-specific antibodies (9).

ninth mutant, M5, contained three alterations: A25T, R87K, and a deletion of D52. Of the nine altered amino acids in the nine mutants, four were conserved in several adenovirus serotypes. Although an exact repetition of the same mutation could be the product of one mutagenic event, the repeated appearance of mutation R81C in M2, M8, and M3, which contained an additional deletion, was probably the result of at least two mutagenic events that were selected in our screening procedure.

In general, the mutations were clustered in four regions containing conserved amino acids: amino acids (aa) 4 to 8 , aa 25 to 29 , aa 48 to 62 , and aa 81 to 87 . Other mutations, previously described as significantly reducing apoptosis, included alterations in aa 9 to $10,21,51,78,88$ to 89,93 to 95 , and $105(7,11)$. Since conflicting results have been reported regarding aa 73 to 75 , they have not been included here. Thus, some of the mutations selected in yeast fall in E4orf4 domains previously identified as involved in induction of apoptosis, and some may define additional domains. A summary of the amino acids whose alterations led to at least a twofold reduction in apoptosis is shown in Fig. 2B. The structural consequences of altering these sites can be understood in detail only when the structure of E4orf4 has been solved. However, since the mutations all cluster in specific regions, they may well affect structural features of the protein and influence protein-protein interactions.

To test whether loss of toxicity in yeast reflects the loss of proapoptotic activities in mammalian cells, the seven different mutant cDNAs obtained in the yeast screen were subcloned into the pcDNA3 vector (Invitrogen), and their ability to induce apoptosis was tested in 293 T cells. Preliminary experiments demonstrated that all of the mutants were expressed in $293 \mathrm{~T}$ cells. However, to obtain levels of expression comparable to those of the wt E4orf4, higher amounts of mutant plasmid DNAs had to be transfected into the cells (results not shown) (Fig. 3A). After having found the conditions for obtaining equal expression of wt and mutant E4orf4 proteins, we used these conditions to test the ability of the mutants to induce apoptosis. The $293 \mathrm{~T}$ cells were transfected with the various constructs together with a vector expressing green fluorescent protein, and nuclei were stained with 4', 6' -diamidino-2-phenylindole (DAPI) at $24 \mathrm{~h}$ posttransfection. The fraction of the transfected cell population that manifested apoptotic morphology of the nucleus was quantified in three independent experiments, as previously described $(9,10,11)$. Figure 3B demonstrates that none of the mutant constructs induced apoptosis to significantly higher levels than the empty vector, whereas wt E4orf4 induced apoptosis in these cells. Thus, E4orf4-induced toxicity in the yeast system is apparently an accurate reflection

A

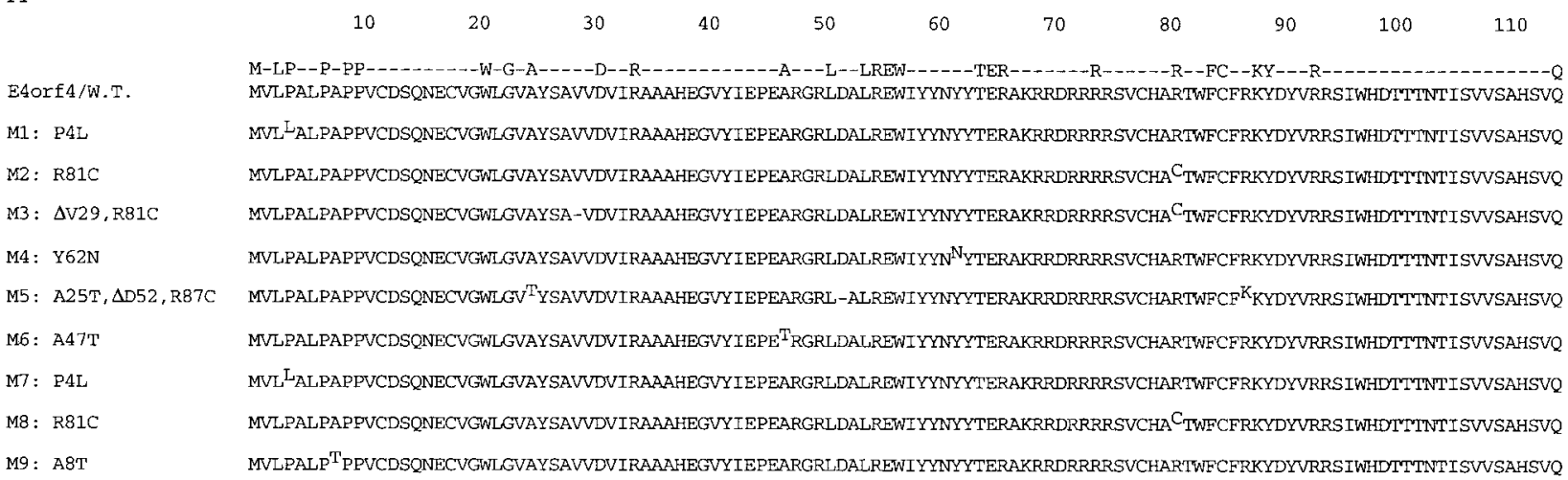

B

50

$60 \quad 70$

80

90

100

110

E40rf4: MVLPALPAPPVCDSQNECVGWLGVAYSAVVDVIRAAAHEGVY IEPEARGRLDALREWIYYNYYTERAKRRDRRRRSVCHARTWECFRKYDYVRRS IWHDTTTITISVVSAHSVQ

FIG. 2. E4orf4 mutations. (A) Sequence of E4orf4 mutants obtained by selection in S. cerevisiae. The mutations are shown in superscript. Amino acids conserved in several adenovirus serotypes are noted at the top of the panel. (B) The amino acids whose alteration led to at least twofold reduced induction of apoptosis by E4orf4 are highlighted in bold type. Conserved amino acids are underlined. The information was collected from three reports $(7,11)$ (Fig. 3). 
A

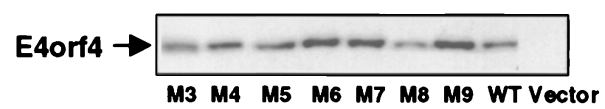

B

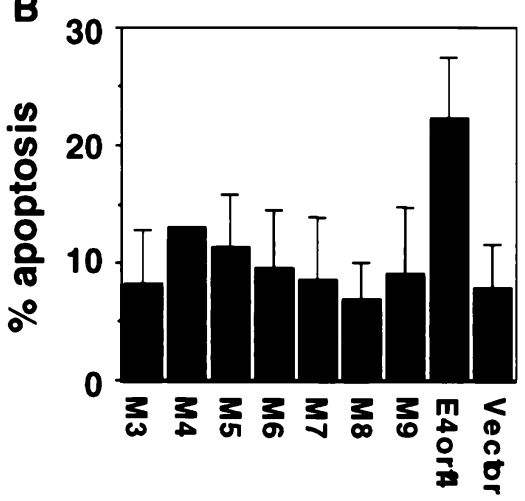

FIG. 3. Induction of apoptosis in $293 \mathrm{~T}$ cells by yeast-selected E4orf4 mutants. (A) E4orf4 and the yeast-selected mutants were cloned in a pcDNA3 vector and expressed in $293 \mathrm{~T}$ cells. The wt E4orf4 was transfected at $1 \mu \mathrm{g}$ of DNA per plate, and the mutants were transfected at $14 \mu \mathrm{g}$ (M7 and M9) or $9 \mu \mathrm{g}$ (all of the rest) per plate. E4orf4 protein levels were analyzed by Western blotting. (B) Using DNA quantities as in panel A, wt E4orf4 and the mutants were cotransfected into $293 \mathrm{~T}$ cells with a plasmid expressing green fluorescent protein. Twenty-four hours posttransfection, nuclei were stained with DAPI (Sigma) and the percentage of apoptosis was determined by counting nuclei with apoptotic morphology in the transfected cell population, identified by the green fluorescence. One hundred nuclei were counted for each point, and the graph shows the average of three experiments. Error bars indicate standard deviations.

of E4orf4-induced apoptosis in mammalian cells and may be utilized in the future for further analysis of the E4orf4 apoptotic pathway.

We next determined whether the mutants retained the ability to bind PP2A. Mutant and wt E4orf4 cDNAs were transfected into $293 \mathrm{~T}$ cells in quantities that assured similar levels of E4orf4 expression (Fig. 4A). E4orf4 proteins were immunoprecipitated from equal amounts of cell extracts, and phosphatase assays were performed as previously described (11). When required, phosphatase activities were normalized to E4orf4 protein levels determined by Western blotting; the results are shown in Fig. 4C. Subsequently, the proteins included in the immune complexes were resolved by sodium dodecyl sulfatepolyacrylamide gel electrophoresis, the presence of the PP2A catalytic subunit was detected on Western blots (Fig. 4A), and binding levels were quantified by densitometry and normalized to E4orf4 levels (Fig. 4B). The results demonstrated that the mutants bound reduced levels (1.8- to 2.5-fold) of PP2A compared with the wt E4orf4 protein. The relative levels of phosphatase activity associated with the mutant proteins were even lower than the relative levels of physical association (three- to sevenfold). This behavior is reminiscent of mutants previously described (R1, R2, and 19) (11), which exhibited decreased phosphatase activity compared to a more efficient binding of PP2A.

In summary, the selection for the nonapoptotic E4orf4 mutants in yeast described here was based on two requirements: loss of E4orf4 toxicity and conservation of wt levels of E4orf4 expression. Our screening procedure yielded several E4orf4 mutants that were deficient in their ability to bind an active PP2A holoenzyme and were unable to induce apoptosis in mammalian cells. These results reinforce the previously demonstrated correlation between diminished association of E4orf4 with an active PP2A holoenzyme and its reduced ability to induce apoptosis $(7,11)$. Our results further suggest that E4orf4induced toxicity in yeast reflects accurately E4orf4-induced apoptosis in mammalian cells, and the yeast system may thus

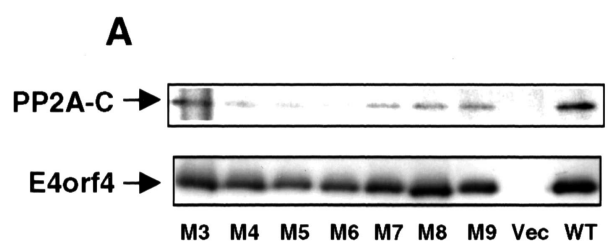

B
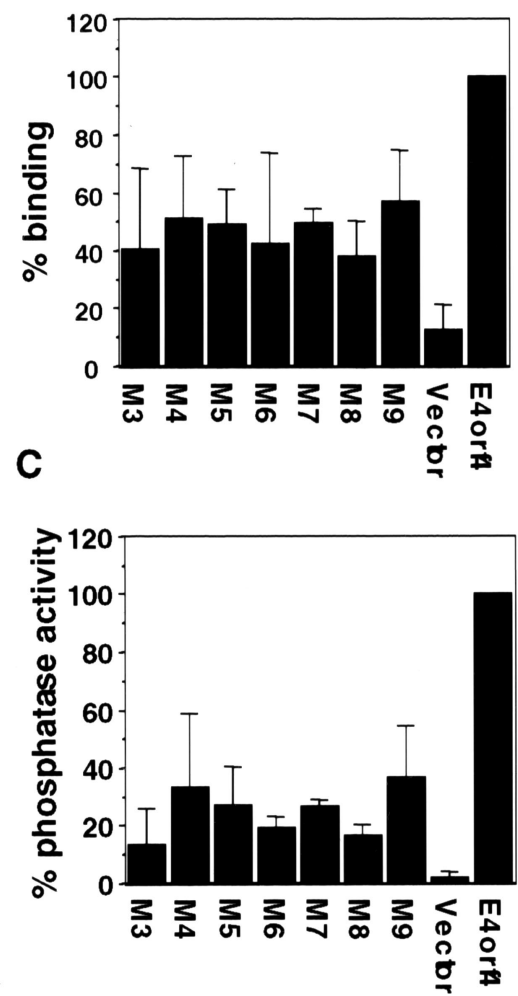

FIG. 4. Association of the yeast-selected, nonapoptotic E4orf4 mutants with PP2A. The wt E4orf4 and mutants and the empty vector (Vec) were transfected into $293 \mathrm{~T}$ cells, as described in the legend for Fig. 3. E4orf4 was immunoprecipitated for $1 \mathrm{~h}$ from equal amounts of protein extracts, and phosphatase assays were carried out as previously described (11). Subsequently, the proteins in the immune complexes were subjected to sodium dodecyl sulfate-polyacrylamide gel electrophoresis, and Western blots were stained with antibodies to PP2A-C and E4orf4. (A) Binding levels were determined by densitometry and normalized to E4orf4 levels. Binding to wt E4orf4 was defined as $100 \%$. (B) Results of four independent experiments measuring PP2A binding levels. Error bars indicate standard deviations. (C) Results of three independent experiments measuring PP2A activity. Error bars indicate standard deviations. 
serve as a powerful tool to genetically dissect the E4orf4 apoptotic pathway.

R. Afifi and R. Sharf contributed equally to this work.

We thank D. Kornitzer for helpful discussions during the course of mutant selection in yeast and for his comments on the manuscript. We also thank all members of our laboratory for technical assistance and advice.

This work was supported by grants from the Israel Science Foundation founded by the Israel Academy of Sciences and Humanities, the Israeli Ministry of Sciences, and from the Fund for the Promotion of Research at the Technion.

\section{REFERENCES}

1. Kanopka, A., O. Muhlemann, S. Petersen-Mahrt, C. Estmer, C. Ohrmalm, and G. Akusjarvi. 1998. Regulation of adenovirus alternative RNA splicing by dephosphorylation of SR proteins. Nature 393:185-187.

2. Kleinberger, T. 2000. Induction of apoptosis by adenovirus E4orf4 protein. Apoptosis 5:211-215.

3. Kleinberger, T., and T. Shenk. 1993. Adenovirus E4orf4 protein binds to protein phosphatase $2 \mathrm{~A}$, and the complex down regulates E1A-enhanced junB transcription. J. Virol. 67:7556-7560.

4. Lavoie, J. N., M. Nguyen, R. C. Marcellus, P. E. Branton, and G. C. Shore. 1998. E4orf4, a novel adenovirus death factor that induces p53-independent apoptosis by a pathway that is not inhibited by zVAD-fmk. J. Cell Biol. 140:637-645.
5. Livne, A., R. Shtrichman, and T. Kleinberger. 2001. Caspase activation by adenovirus E4orf4 protein is cell line specific and is mediated by the death receptor pathway. J. Virol. 75:789-798.

6. Marcellus, R. C., J. N. Lavoie, D. Boivin, G. C. Shore, G. Ketner, and P. E. Branton. 1998. The early region 4 orf4 protein of human adenovirus type 5 induces p53-independent cell death by apoptosis. J. Virol. 72:7144-7153.

7. Marcellus, R. C., H. Chan, D. Paquette, S. Thirlwell, D. Boivin, and P. E. Branton. 2000. Induction of p53-independent apoptosis by the adenovirus E4orf4 protein requires binding to the $\mathrm{B} \alpha$ subunit of protein phosphatase 2A. J. Virol. 74:7869-7877.

8. Müller, U., T. Kleinberger, and T. Shenk. 1992. Adenovirus E4orf4 protein reduces phosphorylation of c-fos and E1A proteins while simultaneously reducing the level of AP-1. J. Virol. 66:5867-5878.

9. Shtrichman, R., and T. Kleinberger. 1998. Adenovirus type 5 E4 open reading frame 4 protein induces apoptosis in transformed cells. J. Virol. 72:2975-2982.

10. Shtrichman, R., R. Sharf, and T. Kleinberger. 2000. Adenovirus E4orf4 protein interacts with both $\mathrm{B} \alpha$ and $\mathrm{B}^{\prime}$ subunits of protein phosphatase $2 \mathrm{~A}$, but E4orf4-induced apoptosis is mediated only by the interaction with $\mathrm{B} \alpha$. Oncogene 19:3757-3765.

11. Shtrichman, R., R. Sharf, H. Barr, T. Dobner, and T. Kleinberger. 1999. Induction of apoptosis by adenovirus E4orf4 protein is specific to transformed cells and requires an interaction with protein phosphatase 2A. Proc. Natl. Acad. Sci. USA 96:10080-10085.

12. Sikorski, R. S., and J. D. Boeke. 1991. In vitro mutagenesis and plasmid shuffling: from cloned gene to mutant yeast, p. 302-318. In C. Guthrie and G. R. Fink (ed.), Methods in enzymology, vol. 194. Guide to yeast genetics and molecular biology. Academic Press, Inc., San Diego, Calif. 